\title{
In vitro Activity of Monoclonal and Recombinant Yeast Killer Toxin-like Antibodies Against Antibiotic-resistant Gram-positive Cocci
}

\author{
S. Conti, ${ }^{1}$ W. Magliani, ${ }^{1}$ S. Arseni, ${ }^{1}$ E. Dieci, R. Frazzi, ${ }^{1}$ A. Salati, ${ }^{1}$ \\ P. E. Varaldo, ${ }^{2}$ and L. Polonelli ${ }^{1}$ \\ ${ }^{1}$ Sezione di Microbiologia, Dipartimento di Patologia e Medicina di Laboratorio, \\ Università degli Studi di Parma, Parma, Italy \\ ${ }^{2}$ Istituto di Microbiologia, Facoltà di Medicina e Chirurgia, Università degli Studi \\ di Ancona, Ancona, Italy
}

Accepted May 18, 2000

\begin{abstract}
Background: Monoclonal (mAbKT) and recombinant single-chain (scFvKT) anti-idiotypic antibodies were produced to represent the internal image of a yeast killer toxin (KT) characterized by a wide spectrum of antimicrobial activity, including Gram-positive cocci. Pathogenic eukaryotic and prokaryotic microorganisms, such as Candida albicans, Pneumocystis carinii, and a multidrug-resistant strain of Mycobacterium tuberculosis, presenting specific, although yet undefined, KT-cell wall receptors (KTR), have proven to be killed in vitro by mAbKT and scFvKT. mAbKT and scFvKT exert a therapeutic effect in vivo in experimental models of candidiasis and pneumocystosis by mimicking the functional activity of protective antibodies naturally produced in humans against KTR of infecting microorganisms. The swelling tide of concern over increasing bacterial resistance to antibiotic drugs gives the impetus to develop new therapeutic compounds against microbial threat. Thus, the in vitro bactericidal activity of mAbKT and scFvKT against gram-positive, drug-
\end{abstract}

resistant cocci of major epidemiologial interest was investigated.

Materials and Methods: mAbKT and scFvKT generated by hybridoma and DNA recombinant technology from the spleen lymphocytes of mice immunized with a KT-neutralizing monoclonal antibody (mAb KT4) were used in a conventional colony forming unit (CFU) assay to determine, from a qualitative point of view, their bactericidal activity against Staphylococcus aureus, S. haemolyticus, Enterococcus faecalis, E. faecium, and Streptococcus pneumoniae strains. These bacterial strains are characterized by different patterns of resistance to antibiotics, including methicillin, vancomycin, and penicillin.

Results: According to the experimental conditions adopted, no bacterial isolate proved to be resistant to the activity of mAbKT and scFvKT.

Conclusions: scFvKT exerted a microbicidal activity against multidrug resistant bacteria, which may represent the basis for the drug modeling of new antibiotics with broad antibacterial spectra to tackle the emergence of microbial resistance.

\section{Introduction}

The spread of bacterial resistance to antibiotics has become a major public health problem over the past decade $(1-3)$. The growing concern

Address correspondence and reprint requests to: Prof. Luciano Polonelli, Sezione di Microbiologia, Dipartimento di Patologia e Medicina di Laboratorio, Università degli Studi di Parma, Viale Antonio Gramsci, 14, 43100 Parma, Italy. Phone 052 1-988885; Fax 052 1-993620; E-mail: lucpol@unipr.it about the future of antibacterial chemotherapy has led to fears of a return to a pre-antibiotic era (4). Resistance control strategies must involve concerted efforts by patients, physicians, the pharmaceutical industry, and the public health community (1). The ensuing years unavoidably will be characterized by strict guidelines for prescriptions and the clinical use of antibiotics, as well as severe restrictions on their use in agriculture, fish farms, and animal 
husbandry $(1,2,5)$. It is, nevertheless, difficult to anticipate to what extent prudent use of antibiotics will really affect the resistant bacteria already present in human and environmental reservoirs. Recent studies suggest that a reduction in the use of antibiotics might be unsuccessful at reversing the high levels of antibiotic resistance currently found in hospitals and communities $(6,7)$. Therefore, the development, not only of new conventional antibiotics, but also of novel compounds and alternative strategies for the battle against bacterial infections is becoming a topical and widely recognized need.

In recent years, we produced antibodies with antibiotic activity in polyclonal, monoclonal, and single-chain recombinant formats. These antibodies bear the internal image of a yeast killer toxin (KT) produced by a selected strain of Pichia anomala and characterized by a wide spectrum of antimicrobial activity, including against Gram-positive bacteria, but with a yet undetermined mechanism of action (8-13). In the related Williopsis mrakii killer system, HM-1 killer toxin kills susceptible strains, presumably through interference with the synthesis of the cell wall $\beta$-1,3-glucan (14).

Like KT, monoclonal (mAbKT) and/or single-chain recombinant (scFvKT) KT-like antibodies were proven to kill Candida albicans, Pneumocystis carinii and multidrug-resistant (MDR) Mycobacterium tuberculosis cells in vitro. They were also therapeutic in experimental models of candidiasis and pneumocystosis (12-16). Although the opsonophagogytic activity monoclonal antibodies in vivo cannot be disregarded, the even more potent therapeutic effect of mAbKT and scFvKT attests to their intrinsic antibiotic activity. mAbKT and scFvKT were shown to be equivalent functionally to animal and human protective antibodies elicited in serum or vaginal fluid by an undetermined, yet specific, KT receptor (KTR) of sensitive microorganisms involved in experimental and natural infections (17).

In this report we present, from a qualitative point of view, the in vitro activity of mAbKT and scFvKT against several Gram-positive cocci with different antibiotic resistance patterns, including methicillin-resistant and borderlinesusceptible staphylococci, vancomycin-resistant enterococci, and penicillin-resistant pneumococci. This study offers a rationale to design new antibiotics with broad antimicrobial spectra against current drug-resistant bacteria.

\section{Materials and Methods}

\section{Bacterial Strains}

The bacterial strains used in this study and their relevant antibiotic susceptibilities are presented in Table 1. All were clinical isolates, except for Staphylococcus aureus LSI-R and S. haemolyticus 221-4, which were stable teicoplanin-resistant clones obtained in population studies from heterogeneously teicoplanin-susceptible clinical isolates (methicillin-resistant the former and methicillin-susceptible the latter). Staphylococcus aureus a3 and a53 were two borderline methicillin-susceptible isolates. Staphylococcus aureus a38 and CVC4 were two methicillinresistant isolates, the latter with reduced susceptibility to teicoplanin. Staphylococcus haemolyticus 7086, SH8, and 615 were teicoplanin-resistant isolates (SH8 and 615 were also methicillin-resistant). Enterococcus faecium LS10 and E. faecalis 2724 were two highly vancomycin- and teicoplanin-resistant VanA isolates. Streptococcus pneumoniae strains 5353 and 143 were penicillin-resistant; whereas, strain 153 was penicillin-intermediate.

\section{$m A b K T$}

The immunoglobulin $M$ (IgM) KT-like mAb (mAb K10) used throughout this study was produced according to a previously described procedure (12). The hybridoma secreting mAb K10 was obtained by the fusion of myeloma cells IA983F and the spleen lymphocytes of rats immunized with the KT-neutralizing $\mathrm{mAb}$ KT4 (18). The hybridoma cells were grown in RPMI 1640 medium (Sigma Chemical Co., St. Louis, MO) with 15\% fetal calf serum (Sigma). mAb K10 was purified from culture supernatants by precipitation with ammonium sulfate and dialysis against phosphate-buffered saline (PBS).

\section{scFvKT}

KT-like scFv (H6) used throughout this study was produced according to the procedure previously described by using the commercial Recombinant Phage Antibody System (Pharmacia Biotech AB, Uppsala, Sweden) (13).

Briefly, mRNA from spleen lymphocytes of mice immunized with the KT-neutralizing mAb KT4 was reverse-transcribed and a scFv antibody library was constructed by cloning the relative antibody genes into a specific phagemid vector. Recombinant phages, pro- 
Table 1. Bacterial test strains and their antibiotic susceptibilities

\begin{tabular}{|c|c|c|c|c|c|}
\hline \multirow[b]{2}{*}{ Strain } & \multicolumn{5}{|c|}{ MIC $(\mu \mathrm{g} / \mathrm{ml})$ of } \\
\hline & Penicillin & Oxacillin & Methicillin & Vancomycin & Teicoplanin \\
\hline Enterococcus faecium LS10 & $\geqslant 128$ & & & $\geqslant 128$ & $\geqslant 128$ \\
\hline E. faecalis 2724 & 4 & & & $\geqslant 128$ & $\geqslant 128$ \\
\hline Staphylococcus aureus a38 & $\geqslant 128$ & $\geqslant 128$ & $\geqslant 128$ & 1 & 1 \\
\hline S. aureus a3 & 64 & 2 & 4 & 0.25 & 0.5 \\
\hline S. aureus a53 & $\geqslant 128$ & 2 & 4 & 0.5 & 0.5 \\
\hline S. aureus CVC4 & $\geqslant 128$ & $\geqslant 128$ & $\geqslant 128$ & 2 & 8 \\
\hline S. aureus LS I-R & $\geqslant 128$ & $\geqslant 128$ & $\geqslant 128$ & 2 & 32 \\
\hline S. haemolyticus SH8 & $\geqslant 128$ & $\geqslant 128$ & $\geqslant 128$ & 2 & 64 \\
\hline S. haemolyticus 221-4 & 0.12 & 0.5 & 1 & 2 & 64 \\
\hline S. haemolyticus 615 & $\geqslant 128$ & $\geqslant 128$ & $\geqslant 128$ & 2 & 32 \\
\hline S. haemolyticus 7086 & 0.12 & 0.25 & 0.25 & 2 & 64 \\
\hline Streptococcus pneumoniae 143 & 4 & & & 0.12 & $\leqslant 0.03$ \\
\hline S. pneumoniae 153 & 1 & & & 0.12 & $\leqslant 0.03$ \\
\hline S. pneumoniae 5353 & 4 & & & 0.25 & $\leqslant 0.03$ \\
\hline
\end{tabular}

duced in a transformed Escherichia coli strain (TGl), were repeatedly panned against mAb KT4 and screened by a conventional ELISA against the same mAb. The selected recombinant phages were used to infect a nonsuppressor E. coli HB2151 strain to produce soluble recombinant $S c F v$ that were purified by affinity chromatography using an anti-E Tag Nhydroxysuccinimide activated Sepharose column (Pharmacia Biotech AB).

\section{In vitro Bacterial Killing Assay}

The qualitative killing assay against the bacterial isolates investigated in this study was carried out by using mAbKT against all of the bacterial isolates studied, and scFvKT against four representative bacterial strains (E. faecium LS10, E. faecalis 2724, S. aureus a38, and S. pneumoniae 5353) in a conventional colony forming unit (CFU) assay. Bacterial isolates were grown on Mueller Hinton agar or $10 \%$ horse blood agar plates at $37^{\circ} \mathrm{C}$ for $24 \mathrm{hr}$. Bacterial cells from isolated colonies were suspended in saline to an optical density of 0.5 McFarland and then diluted in Mueller Hinton broth (1:1000 Enterococcus isolates, 1:500 S. haemolyticus isolates, 1:2000 S. aureus isolates, and 1:1000 $S$. pneumoniae isolates, respectively). $10 \mu \mathrm{l}$ of this suspension, containing approximately $2-3 \times 10^{3}$ viable bacterial cells, were added with $100 \mu \mathrm{g}$ of purified mAb K10 or $10 \mu \mathrm{g}$ of purified ScFv H6 in a total volume of $100 \mu \mathrm{l}$. For S. pneumoniae isolates, $1 \%$ Supplement B (Difco Laboratories, Detroit, MI) also was added. All the bacterial suspensions were incubated for $5 \mathrm{hr}$ at $37^{\circ} \mathrm{C}$. As controls, in the in vitro experiments of antimicrobial activity, we used an irrelevant, isotype-matched, commercially available mAb (ABPC 22; Sigma Chemical Co.) and an irrelevant ScFv anti-idiotypic antibody (antiId) previously described (19). As a further control, a similar inoculum of bacterial cells was also added to mAbKT or ScFvKT preincubated overnight at $4^{\circ} \mathrm{C}$ with $100 \mu \mathrm{g}$ of mAb KT4. After incubation with the respective reagents, the bacterial cells were dispensed in Petri dishes and covered with molten Mueller Hinton agar that was allowed to solidify. The plates were then incubated at $37^{\circ} \mathrm{C}$ and observed after $48 \mathrm{hr}$ for bacterial CFU enumeration. Each experiment was performed in triplicate and the results were calculated as a mean. In one circumstance, the CFU assay was also carried out by plating $10 \mu \mathrm{l}$ of bacterial cell suspensions ( $S$. aureus a38) after incubation with scFv $\mathrm{H} 6$ and appropriate controls for $24 \mathrm{hr}$.

\section{Results}

The bactericidal activity of mAb K10 and scFv H6 against the antibiotic-resistant Grampositive cocci qualitatively investigated in this 
Table 2. Antibacterial antibiotic activity of mAb K10

\begin{tabular}{lc}
\hline Microorganism & \% Inhibition \pm Standard Deviation \\
\hline Enterococcus faecium LS10 & $88.83 \pm 8.78$ \\
E. faecalis 2724 & $94.40 \pm 3.91$ \\
Staphylococcus aureus a38 & $89.27 \pm 9.42$ \\
S. aureus a3 & $85.96 \pm 4.45$ \\
S. aureus a53 & $89.59 \pm 4.32$ \\
S. aureus CVC4 & $82.12 \pm 7.79$ \\
S. aureus LS1-R & $89.05 \pm 5.69$ \\
S. haemolyticus SH8 & $77.79 \pm 7.47$ \\
S. haemolyticus 221-4 & $48.52 \pm 6.50$ \\
S. haemolyticus 615 & $75.02 \pm 2.74$ \\
S. haemolyticus 7086 & $79.82 \pm 5.25$ \\
Streptococcus pneumoniae 143 & $85.29 \pm 2.34$ \\
S. pneumoniae 153 & $93.93 \pm 4.42$ \\
S. pneumoniae 5353 & $89.99 \pm 5.99$ \\
\hline
\end{tabular}

study is presented in Tables 2 and 3, respectively. The results are expressed as \% inhibition, compared with the appropriate control. The bactericidal activity of mAbKT and scFvKT was significantly neutralized by mAb KT4 (data not shown). Figure 1 shows the appearance of the killing and neutralization assay against a representative bacterial isolate $(S$. aureus a38) after $24 \mathrm{hr}$ of incubation with $\mathrm{scFv}$ H6 and appropriate controls. Notably, the bacterial colonies surviving the activity of mAbKT and scFvKT should not be considered as resistant, since they behaved just as sensitively when tested in another CFU assay performed under the same experimental conditions (data not shown).

\section{Discussion}

Among antibiotic-resistant bacteria, Gram-positive cocci have become predominant over the past two decades for their ability of acquiring resistance. This is followed by the horizontal transfer of resistance genes and the clonal expansion and spread of resistant clones $(2,20)$. Notable examples are methicillin-resistant staphylococci and vancomycin-resistant enterococci among nosocomial pathogens, and penicillinresistant pneumococci among communityacquired pathogens. Most methicillin-resistant staphylococci are insensitive to many other conventional antibiotics, thereby requiring the use of glycopeptide antibiotics. Unfortunately, vancomycin treatment failures have been reported in infections caused by methicillinresistant strains of $S$. aureus (22-23), S. haemolyticus $(24,25)$, and $S$. epidermidis (26), with decreased heterogeneous susceptibility to glycopeptides. Borderline methicillin-susceptible (or borderline-resistant) $S$. aureus strains have been associated with widespread nosocomial infections (27). Vancomycin-resistant enterococci, which have become major nosocomial

Table 3. Antibacterial antibiotic activity of scFv $\mathrm{H6}$

\begin{tabular}{lc}
\hline Microorganism & \% Inhibition \pm Standard Deviation \\
\hline Enterococcus faecium LS 10 & $\mathbf{8 2 . 8 8} \pm \mathbf{1 . 8 8}$ \\
Enterococcus faecalis 2724 & $94.61 \pm 0.06$ \\
Staphylococcus aureus a38 & $61.63 \pm 4.06$ \\
Streptococcus pneumoniae 5353 & $89.16 \pm 1.90$ \\
\hline
\end{tabular}




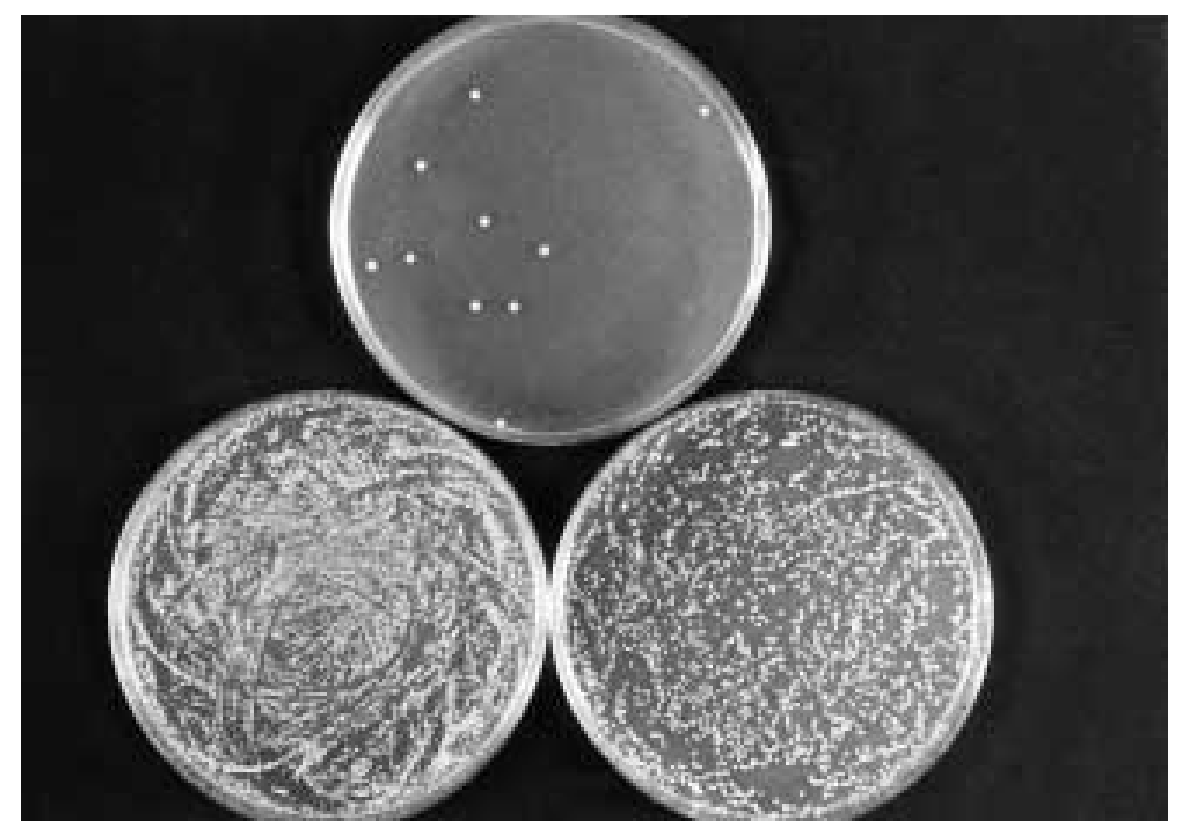

Fig. 1. Effect of single chain fragment variable yeast killer toxin-like recombinant anti-idiotypic (antiId) antibodies (scFvKT) on the growth of Staphylococcus aureus a 38 cells in a colony forming

pathogens, particularly in the U.S.A. $(28,29)$, are often resistant to multiple antibiotics and have been regarded as a classical example of the impact of antimicrobial drug resistance on therapeutic options (30). Moreover, vancomycin resistance can pass from enterococci to other pathogens. Natural spread has been reported among enterococci (32) and other Grampositive bacteria (32-34).

In pneumococci, once among the most highly penicillin-susceptible bacteria, penicillin resistance is now a global problem. Further concern with penicillin-resistant pneumococci currently arises from their increasing resistance to other antimicrobial agents, including third-generation cephalosporins and macrolides $(19,35)$, and from the emergence of tolerance to a number of bactericidal antibiotics, including vancomycin, $\beta$-lactams, aminoglycosides, and quinolones (36). Against such increasingly antibiotic-resistant Gram-positive cocci, antimicrobial research, which until recently has been ahead of the resistance race, must increase its committment for the search of innovative antibiotics. However, continuous modification of conventional drugs seems unlikely to succeed in the long-term fight against bacteria.

Natural short-chain peptides produced by a number of animals as defence mechanisms unit (CFU) assay. Standardized bacterial inocula treated with: scFvKT H6 (top); irrelevant scFv antiIds (lower left); scFvKT H6 neutralized with mAb KT4 (lower right).

have been under study for their broad spectrum of antimicrobial action (37-39). Natural antibiotic peptides usually act by damaging the bacterial cell membrane, but regretfully, they can also damage mammalian cells. In our studies, we demonstrated that monoclonal and recombinant anti-idiotypic antibodies, bearing the internal image of a yeast killer toxin recognized to be active against Gram-positive cocci, may be therapeutic in experimental models of candidiasis and pneumocystosis without the induction of toxic effects $(13,16)$. Even though the mechanism of action is still undetermined and currently under study, it is argued, from the immunofluorescence studies, that it can rely on the interaction with an uncharacterized trans-phyletic cell wall KTR shared by taxonomically unrelated microorganisms. This speculation is corroborated by the findings of functionally equivalent and therapeutic human natural anti-KTR killer antibodies in individuals undergoing repeated infections with KTR-bearing microorganisms (17).

The finding of the microbicidal activity of monoclonal and recombinant KT-like antibodies, which had proven to be killing in vitro and/or in vivo C. albicans, $P$. carinii, and MDR $M$. tuberculosis (12-16), against drug-resistant staphylococci, enterococci, and pneumococci make engineered antibody derivatives particularly 
attractive as therapeutic agents for currently untreatable microbial infections. It is noteworthy that smaller scFv H6 displayed at lower concentrations an antibacterial activity similar to that of higher molecular sized IgM mAb K 10 and that prolonged incubation with microbicidal antibodies may result in a remarkable increase in the rate of killing. The determined sequence of the variable region of the heavy and light chains of ScFvKT should make possible the engineering of new molecules characterized by an unexhausted mechanism of antibiotic activity.

\section{Acknowledgments}

Ministero della Sanità, Istituto Superiore di Sanità, Programma Nazionale di Ricerca sull'AIDS-1998, Accordo di Collaborazione Scientifica n. 50B.32, and Ministero dell'Università e della Ricerca Scientifica e Tecnologica, Programmi di Ricerca Scientifica di Interesse Nazionale-1999.

\section{References}

1. Kunin CM. (1993) Resistance to antimicrobial drugs-a worldwide calamity. Ann. Int. Med. 118: 557-561.

2. Tomasz A. (1994) Multiple antibiotic-resistant pathogenic bacteria. A report on the Rockfeller University workshop. N. Engl. J. Med. 330: 12471251.

3. Gold HS, Moellering RC. (1996) Antimicrobial drug-resistance. N. Engl. J. Med. 335: 1445-1453.

4. Cohen ML. (1992) Epidemiology of drug resistance: implications for a post-antimicrobial era. Science 257: 1050-1055.

5. Hawkey PM. (1998) Action against antibiotic resistance: no time to lose. Lancet 351: 12981299.

6. Spratt BG. (1996) Antibiotic resistance: counting the cost. Curr. Biol. 6: 1219-1221.

7. Biörkman J, Hughes D, Andersson DI. (1998) Virulence of antibiotic-resistant Salmonella typhimurium. Proc. Natl. Acad. Sci. U.S.A. 95: 39493953.

8. Polonelli L, Morace G. (1986) Reevaluation of the yeast killer phenomenon. J. Clin. Microbiol. 24: 866-869.

9. Polonelli L, Conti S, Gerloni M, et al. (1991) "Antibiobodies": antibiotic-like anti-idiotypic antibodies. J. Med. Vet. Mycol. 29: 235-242.

10. Polonelli L, Lorenzini R, De Bernardis F, et al. (1993) Idiotypic vaccination: immunoprotection mediated by anti-idiotypic antibodies with antibiotic activity. Scan. J. Immunol. 37: 105- 110.

11. Polonelli L, De Bernardis F, Conti S, et al. (1994) Idiotypic intravaginal vaccination to protect against candidal vaginitis by secretory, yeast killer toxin-like antiidiotypic antibodies. J. Immunol. 152: 3175-3182.

12. Polonelli L, Séguy N, Conti S, et al. (1997) Monoclonal yeast killer toxin-like candidacidal antiidiotypic antibodies. Clin. Diagn. Lab. Immunol. 4: 142-146.

13. Magliani W, Conti S, De Bernardis F, et al. (1997) Therapeutic potential of antiidiotypic single chain antibodies with yeast killer toxin activity. Nature Biotechnol. 15: 155-158.

14. Magliani W, Conti S, Gerloni M, Bertolotti D, Polonelli L. (1997) Yeast killer systems. Clin Microbiol Rev. 10: 369-400.

15. Conti S, Fanti F, Magliani W, et al. (1998) Mycobactericidal activity of human natural, monoclonal, and recombinant yeast killer toxin-like antibodies. J. Infect. Dis. 177: 807-811.

16. Séguy N, Polonelli L, Dei-Cas E, Cailliez JC. (1998) Perspectives in the control of Pneumocystis infections by using Pichia anomala killer toxinlike antiidiotypic antibodies. FEMS Immunol. Med. Microbiol. 22: 145-149.

17. Polonelli L, De Bernardis F, Conti S, et al. (1996) Human natural yeast killer toxin-like candidacidal antibodies. J. Immunol. 156: 1880-1885.

18. Polonelli L, Morace G. (1987) Production and characterization of yeast killer toxin monoclonal antibodies. J. Clin. Microbiol. 25: 460-462.

19. Magliani W, Polonelli L, Conti S, et al. (1998) Neonatal mouse immunity against group $B$ streptococcal infection by maternal vaccination with recombinant antiidiotypes. Nature Med. 4: 705-709.

20. Moellering RC. (1998) Introduction: problems with antimicrobial resistance in gram-positive cocci. Clin. Infect. Dis. 26: 1177-1178.

21. Centers for Disease Control and Prevention. (1997) Update: Staphylococcus aureus with reduced susceptibility to vancomycin-United States. MMWR 46: 813-815.

22. Hiramatsu K, Aritaka N, Hanaki H, et al. (1997) Dissemination in Japanese hospitals of strains of Staphylococcus aureus heterogeneously resistant to vancomycin. Lancet 350: 1670-1673.

23. Sieradzki K, Roberts RB, Haber SW, Tomasz A. (1999) The development of vancomycin resistance in a patient with methicillin-resistant Staphylococcus aureus infection. N. Engl. J. Med. 340: 517-523.

24. Schwalbe RS, Stapleton JT, Gilligan PH. (1987) Emergence of vancomycin resistance in coagulase-negative staphylococci. N. Engl. J. Med. 316: 927-931.

25. Veach LA, Pfaller MA, Barrett M, Koontz FP, Wenzel RP. (1990) Vancomycin resistance in Staphylococcus haemolyticus causing colonization 
and bloodstream infection. J. Clin. Microbiol. 28: 2064-2068.

26. Sieradzki K, Roberts RB, Serur D, Hargrave J, Tomasz A. (1998) Recurrent peritonitis in a patient on dialysis and prophylactic vancomycin. Lancet 351: 880-881.

27. McMurray LW, Kernodle DS, Barg N. (1990) Characterization of a widespread strain of methicillin-susceptible Staphylococcus aureus associated with nosocomial infections. J. Infect. Dis. 162: 759-762.

28. Murray BE. (1997) Vancomycin-resistant enterococci. Am. J. Med. 102: 284-293.

29. Moellering RC. (1998) Vancomycin-resistant enterococci. Clin. Infect. Dis. 26: 1196-1199.

30. Moellering RC. (1991) The enterococcus: a classic example of the impact of antimicrobial resistance on therapeutic options. J. Antimicrob. Chemother. 28: 1-12.

31. Dutka-Malen S, Blaimont B, Wauters G, Courvalin P. (1994) Emergence of high-level resistance to glycopeptides in Enterococcus gallinarum and Enterococcus casseliflavus. Antimicrob. Agents Chemother. 38: 1675-1677.

32. French G, Abdulla Y, Heathcock R, Poston S, Cameron J. (1992) Vancomycin resistance in south London. Lancet 339: 818-819.

33. Fontana R, Ligozzi M, Pedrotti C, Padovani EM, Cornaglia G. (1997) Vancomycin-resistant Bacil- lus circulans carrying the vanA gene responsible for vancomycin resistance in enterococci. Eur. $J$. Clin. Microbiol. Infect. Dis. 16: 473-474.

34. Poyart C, Pierre C, Quesne G, Pron B, Berche P, Trieu-Cuot P. (1997) Emergence of vancomycin resistance in the genus Streptococcus: characterization of a vanB transferable determinant in Streptococcus bovis. Antimicrob. Agents Chemother. 41: 24-29.

35. Tomasz A. (1997) Antibiotic resistance in Streptococcus pneumoniae. Clin. Infect. Dis. 24 (Suppl. 1): S85-88.

36. Novak R, Henriques B, Charpentier E, Normark S, Tuomanen E. (1999) Emergence of vancomycin tolerance in Streptococcus pneumoniae. $\mathrm{Na}$ ture 399: 590-593.

37. Boman HG. (1995) Peptide antibiotics and their role in innate immunity. Annu. Rev. Immunol. 13: 61-92.

38. Hancock RE. (1997) Peptide antibiotics. Lancet 349: 418-422.

39. Lehrer RI, Lichtenstein AK, Ganz T. (1993) Defensins: antimicrobial and cytotoxic peptides of mammalian cells. Annu. Rev. Immunol. 11: 105-128.

40. Nicolas P, Mor A. (1994) Peptides as weapons against microorganisms in the chemical defense system of vertebrates. Annu. Rev. Microbiol. 49: 277-304. 